Pacific Journal of Mathematic 


\section{TOTAL POSITIVITY AND REPRODUCING KERNELS}

\section{JACOB BURBEA}

In this paper we investigate the relationship between total positivity and reproducing kernels. We extend the notion of total positivity to domains in the complex plane. In doing so, we also give a geometrical interpretation to certain Wronskians of reproducing kernels. These geometrical quantities are connected to Gaussian curvatures of Kähler metrics induced by these kernels. For simply-connected domains these curvatures are negative constants, thereby showing that the kernels are totally positive and moreover yielding an efficient method for computing the relevant determinants. In general, the reproducing kernels of multiplyconnected domains are not totally positive.

The motivation for this work stems from the work of Karlin [7] which deals with "optimal" quadrature formulas.

Let $H$ be a Hilbert space of functions analytic in a plane domain $D$ and possessing a reproducing kernel $K(z, \bar{t}), z, t \in D$. Let $L \in H^{*}$, where $H^{*}$ is the dual of $H$. A subset $Q$ of $H^{*}$ is specified and a member $Q \in \mathbb{Q}$ is called a quadrature formula. To each $Q \in \mathbb{Q}$ is associated a remainder functional $R_{Q}=L-Q$. An optimal quadrature formula, if it exists, is any member $Q^{*} \in \mathbb{Q}$ satisfying

$$
\left\|R_{Q^{*}}\right\|=\operatorname{Inf}_{Q \in \mathscr{Q}}\left\|R_{Q}\right\|=\operatorname{Inf}_{\hat{Q} \in \hat{Q}}\|\hat{L}-\hat{Q}\|,
$$

where $\hat{L}$ is the representor of $L$ in $H^{*}$ and $\hat{\mathscr{Q}}$ is the set of all representors of functionals in $\mathscr{Q}$. Since $H$ has a reproducing kernel $K(z, \bar{t})$ it follows (see [5], pp. 318-319) that $\hat{L}(t)=\overline{L_{z}(z, \bar{t})}$ and

$$
\left\|R_{Q^{*}}\right\|^{2}=\left(L_{t}-Q_{t}^{*}\right) \overline{\left(L_{z}-Q_{z}^{*}\right) K(z, \bar{t})} .
$$

(The subscript in $L_{z}$ indicates that $t$ is held fixed and $L$ is applied to $K(z, \bar{t})$ as a function of $z$.)

More specifically, let $\gamma$ be a rectifiable curve lying in $D$ and specify

$$
L(f)=\int_{r} f(z) w(z) d z, \quad f \in H,
$$

where $w(z)$ is an integrable function on $\gamma$. Consider

$$
\mathscr{Q}_{n}=\left\{Q \in H^{*}: Q(f)=\sum_{k=1}^{n} \alpha_{k} f\left(t_{k}\right),\left(t_{k}\right)_{1}^{n} \subset D,\left(\alpha_{k}\right)_{1}^{n} \subset C\right\},
$$


where $n$ is fixed. (The knots $\left(t_{k}\right)_{1}^{n}$ are free variables.) $L$ is of course in $H^{*}$ and its representor $\hat{L}$ is given by

$$
\hat{L}(t)=\int_{r} K(t, \bar{z}) \overline{w(z) d} \bar{z}, \quad t \in D .
$$

Therefore, if $Q \in \mathbb{Q}_{n}$, then

$$
\left\|R_{Q}\right\|=\left\|\int_{r} K(t, \bar{z}) \overline{w(z) d} \bar{z}-\sum_{k=1}^{n} \bar{\alpha}_{k} K\left(t, \bar{t}_{k}\right)\right\|_{(t)},
$$

and

$$
\operatorname{Inf}_{Q \in \mathscr{C}_{n}}\left\|R_{Q}\right\|=\operatorname{Inf}\left\{\left\|R_{Q}\right\|:\left(\alpha_{k}\right)_{1}^{n} \subset C,\left(t_{k}\right)_{1}^{n} \subset D\right\} .
$$

Then the problem of finding the optimal quadrature formula, as described above, is closely related to whether the sequence $\left\langle K\left(t, \bar{t}_{k}\right)\right\rangle_{k=1}^{n}$, for any choice of the $n$ knots $\left(t_{k}\right)_{1}^{n}$, forms an extended complete Tchebycheff system on $D$ (see definition below) or not ([7], [8]). Therefore, the optimality of a quadrature formula is closely connected to the notion of total positivity of $K(z, \bar{t})$ on $D$.

Here, we extend the notion of total positivity to the complex case. By doing so, we also give a geometrical interpretation to certain Wronskians of reproducing kernels. These geometrical quantities are connected to Gaussian curvatures of Kähler metrics induced by the reproducing kernels.

When $D$ is simply-connected (that is when the automorphism group of $D$ is transitive) these curvatures are negative constants, thereby proving that the kernels are totally positive and moreover yielding an efficient method for computing the relevant determinants. In general, the reproducing kernels of multiply-connected domains are not totally positive.

1. Total positivity. We introduce some definitions and notation from the theory of total positivity. We shall use Karlin's book [6] (especially pp. 11-49) as basic reference.

Definition 1. A sequence of real-valued continuous functions $\phi_{1}(x), \phi_{2}(x), \cdots, \phi_{n}(x)$ is said to constitute a Tchebycheff system (T-system) on $[a, b]$ if

$$
\operatorname{det}\left\|\phi_{i}\left(x_{j}\right)\right\|_{i, j=1}^{n}>0
$$

for all $a \leqq x_{1}<x_{2}<\cdots<x_{n} \leqq b$. The sequence is called a complete 
T-system (CT-system) on $[a, b]$ if $\phi_{1}, \cdots, \phi_{k}$ is a $T$-system for $k=1$, $\cdots, n$. The sequence is called a Descartes system ( $D$-system) on $[a, b]$ if $\phi_{\alpha_{1}}, \phi_{\alpha_{2}}, \cdots, \phi_{\alpha_{k}}$ is a $C T$-system for each $1 \leqq \alpha_{1}<\alpha_{2}<\cdots<\alpha_{k} \leqq n$.

Let $D$ be a domain in the complex plane, and let $\phi_{0}, \phi_{1}, \cdots$, $\phi_{m-1} \in \mathscr{C}^{(m-1)}(D)$. The Wronskian of $\phi_{0}, \phi_{1}, \cdots, \phi_{m-1}$ is given by

$$
W\left[\dot{\phi}_{0}(z), \cdots, \dot{\phi}_{m-1}(z)\right]=\operatorname{det}\left\|\frac{\partial^{k} \dot{\phi}_{j}}{\partial z^{k}}\right\|_{k, j=0}^{m-1} .
$$

DEFINITION 2. A sequence of complex-valued functions $\phi_{0}(z), \phi_{1}(z)$, $\cdots, \phi_{m-1}(z)$ of class $\mathscr{C}^{(m-1)}(D)$ is called an extended complete Tchebycheff system $\left(E C T\right.$-system) on $D$ if $W\left[\phi_{0}(z), \cdots, \phi_{k-1}(z)\right] \neq 0$ for $k=1, \cdots$, $m$ and for all $z \in D$.

This definition is an extension of the usual definition of a sequence of real-valued functions $\phi_{0}(x), \phi_{1}(x), \cdots, \phi_{m-1}(x)$ on the interval $[a, b]$. If $\phi_{0}, \phi_{1}, \cdots, \phi_{m-1}$ is ECT on $[a, b]$, then (see [6], p. 52) $\phi_{0}, \phi_{1}, \cdots, \phi_{m-1}$ is $\mathrm{CT}$ on $[a, b]$.

Definition 3. A real-valued function $K(x, y)$ defined on $[a, b] \times$ $[a, b]$ is said to be totally positive (abbreviated TP) on $[a, b] \times[a, b]$ if, for all $a \leqq x_{1}<x_{2}<\cdots<x_{m} \leqq b, a \leqq y_{1}<y_{2}<\cdots<y_{m} \leqq b, m=$ $1,2, \cdots$, we have the inequalities

$$
K\left(\begin{array}{l}
x_{1}, x_{2}, \cdots, x_{m} \\
y_{1}, y_{2}, \cdots, y_{m}
\end{array}\right)=\operatorname{det}\left\|K\left(x_{i}, y_{i}\right)\right\|_{i, j=1}^{m}>0
$$

Definition 4. Let $K(z, \bar{t})$ be of class $\mathscr{C}^{\infty}(D)$ in the two complex variables $z, t \in D . K(z, \bar{t})$ is said to be extended $T P(E T P)$ on $D$ if, for $m=1,2, \cdots$, we have the inequalities

$$
K^{*}(\overbrace{\left.\begin{array}{l}
z, \cdots, z \\
\bar{t}, \cdots, \bar{t}
\end{array}\right)}^{m}=\operatorname{det}\left\|\frac{\partial^{k+j-2}}{\partial z^{k-1} \partial \bar{t}^{j-1}} K(z, \bar{t})\right\|_{k, j=1}^{m} \neq 0 .
$$

Again this definition extends the usual definition to the complex case. If $D$ contains the real interval $[a, b]$, and $K(z, \bar{t})$ is real-valued on $[a, b] \times[a, b]$ and ETP on $[a, b] \times[a, b]$ then (see [6], p. 55) $K(z, \bar{t})$ is $\mathrm{TP}$ on $[a, b] \times[a, b]$.

2. Reproducing kernels. Let $H$ be a Hilbert space of analytic functions in the domain $D$ with the inner product $($,$) and such that$ all polynomials are contained in $H$. In addition, we assume that for each $t \in D$ the linear functional $L_{t}$, defined by $L_{t} f=f(t), f \in H$, is bounded on $H$. According to [1], $H$ possesses a unique reproducing 
kernel $K(z, \bar{t})$ with the following properties:

(i) for each $t \in D, K(, \bar{t}) \in H$;

(ii) for each $t \in D$ and each $f \in H, f(t)=(f, K(, \bar{t}))$.

This kernel satisfies the following lemmas (compare also [1]).

Lemma 1. The form $\sum_{i, j=1}^{n} K\left(t_{i}, \bar{t}_{j}\right) \xi_{i} \bar{\xi}_{j}$ is positive definite for all $t_{1}, \cdots, t_{n} \in D$. In particular, $K(t, \bar{t})>0$ and $\left.K(z, \bar{t})=\overline{K(t, \bar{z}}\right), z, t \in D$.

Lemma 2. $K(z, \bar{t})$ is analytic in $(z, \bar{t})$ for $(z, t) \in D \times D$.

LEMMA 3. If $f_{n} \rightarrow f$ in $H$ then $f_{n} \rightarrow f$ uniformaly on compacta of $D$.

Proof. We have

$$
\left|f_{n}(t)-f(t)\right|=\left|\left(f_{n}-f, K(, \bar{t})\right)\right| \leqq\left\|f_{n}-f\right\| K(t, \bar{t})^{1 / 2} .
$$

The lemma now follows from Lemma 2.

\section{LemMa $4 . \quad H$ is a separable Hilbert space.}

Proof. Let $\left(t_{n}\right)$ be a dense sequence in $D$ and consider the sequence of functions $\psi_{n}(z)=K\left(z, \bar{t}_{n}\right), n=1,2, \cdots$. Then $\left(\psi_{n}\right) \subset H$ and since the polynomials are contained in $H$ this sequence is linearly independent. Moreover, if $\left(f, \psi_{n}\right)=0, n=1,2, \cdots, f \in H$, then $f=0$.

Let $\left(\phi_{n}\right)$ be an orthonormal basis of $H$. Then

$$
K(z, \bar{t})=\sum_{n=1}^{\infty} \Phi_{n}(z) \overline{\phi_{n}(t)},
$$

where, according to the previous lemmas, the infinite sum on the right is independent of the choice of the basis and it converges absolutely and uniformly on compacta of $D$.

We need the following lemma which is, to a certain extent, a reformulation of the Cauchy-Binet formula (see [6], p. 1).

Let $\left(a_{n, k}\right)$ and $\left(b_{n, k}\right)\left(n_{\infty}=1,2, \cdots ; k=1,2, \cdots, m\right)$ be two infinite matrices such that $\sum_{n=1}^{\infty}\left|a_{n, k} b_{n, j}\right|<\infty, 1 \leqq k, j \leqq m$. Let

$$
\left(a_{k}, b_{j}\right) \equiv \sum_{n=1}^{\infty} a_{n, k} b_{n, j}, 1 \leqq k, j \leqq m
$$

and

$$
D_{1}^{\mu_{1} \ldots \mu_{m}} \mu_{m}(a)=\operatorname{det}\left\|\alpha_{\mu_{i}, j}\right\|_{i, j=1}^{m} .
$$

We have: 


\section{LEMMA 5.}

$$
\operatorname{det}\left\|\left(a_{i}, b_{j}\right)\right\|_{i, j=1}^{m}=\sum_{\mu_{1}<\cdots<\mu_{m}} D_{1 \cdots}^{\prime \prime \prime \cdots \mu^{\prime}} m(a) D_{1 \cdots m}^{\mu_{1} \cdots \mu^{\prime} m}(b) .
$$

THEOREM 1. Suppose D contains $[a, b]$ and $\left(\dot{\phi}_{k}\right)_{k=1}^{\infty}$ is an orthonormai basis of $H$. If $\left(\dot{\phi}_{k}\right)_{k=1}^{n}, n=1,2, \cdots$, forms a D-system on $[a, b]$, then $K(z, \bar{t})$ is TP on $[a, b] \times[a, b]$.

Proof. Using Lemma 5, we have

$$
\begin{aligned}
& K\left(\begin{array}{l}
x_{1}, \cdots, x_{n} \\
y_{1}, \cdots, y_{n}
\end{array}\right)=\left|\begin{array}{l}
\sum_{\mu_{1}=1}^{\infty} \phi_{\mu_{1}}\left(x_{1}\right) \dot{\phi}_{\mu_{1}}\left(y_{1}\right) \cdots \sum_{\mu_{1}=1}^{\infty} \phi_{\mu_{1}}\left(x_{1}\right) \dot{\phi}_{\mu_{1}}\left(y_{n}\right) \\
\sum_{\mu_{n}=1}^{\infty} \dot{\rho}_{\mu_{n}}\left(x_{n}\right) \dot{\phi}_{\mu_{n}}\left(y_{1}\right) \cdots \sum_{\mu_{n}=1}^{\infty} \phi_{\mu_{n}}\left(x_{n}\right) \dot{\phi}_{\mu_{n}}\left(y_{n}\right)
\end{array}\right| \\
& =\sum_{\mu_{1}<\cdots<\mu_{n}} \operatorname{det}\left\|\phi_{\mu_{i}}\left(x_{j}\right)\right\|_{i, j=1}^{n} \operatorname{det}\left\|\dot{\phi}_{\mu_{i}}\left(y_{j}\right)\right\|_{i, j=1}^{n}
\end{aligned}
$$

$a \leqq x_{1}<\cdots<x_{n} \leqq b, a \leqq y_{1}<\cdots<y_{n} \leqq b, n=1,2, \cdots$. The last sum is strictly positive by assumption, thereby proving the theorem.

A somewhat converse of this theorem is contained in the following:

Theorem 2. Let $[a, b]$ be contained in $D$ and suppose $K(z, \bar{t})$ is TP on $[a, b] \times[a, b]$. Then:

(1) there exists a basis for $H_{,}\left(\psi_{k}\right)_{k=1}^{\infty}$ such that $\left(\psi_{k}\right)_{k=1}^{n}, n=1$, $2, \cdots$, forms a D-system on $[a, b]$

(2) there exists an orthonormal basis $\left(\dot{\phi}_{k}\right)_{k=1}^{\infty}$ in $H$ such that $\left(\dot{\phi}_{k e}\right)_{k=1}^{n}, n=1,2, \cdots$, forms a CT-system on $[a, b]$.

Proof. Define

$$
\theta_{n}=b-\frac{b-a}{n(b-a)+1}, \quad n=1,2, \cdots
$$

Note that

$$
a<\theta_{1}<\theta_{2}<\cdots<\theta_{n}<\cdots<b, \quad \lim _{n \rightarrow \infty} \theta_{n}=b \in D .
$$

Next, define

$$
\psi_{k}(z)=K\left(z, \theta_{k}\right), z \in D, \quad k=1,2, \cdots .
$$

Clearly, $\left(\psi_{k}\right)$ so defined is linearly independent. Further, suppose there is an $f \in H$ such that $\left(f, \psi_{k}\right)=0, k=1,2, \cdots$. Thus $f\left(\theta_{k}\right)=$ 0 , with $\lim _{k \rightarrow \infty} \theta_{k} \in D$ and hence $f=0$. Moreover, $\left(\psi_{\alpha_{k}}\right)_{k=1}^{n}, 1 \leqq \alpha_{1}<$ $\alpha_{2}<\cdots<\alpha_{n} \leqq m$, is $\mathrm{CT}$ on $[a, b]$. In fact, for $a \leqq t_{1}<t_{2}<\cdots<t_{n} \leqq b$,

$$
\operatorname{det}\left\|\left.\psi_{\alpha_{k}}\left(t_{j}\right)\right|_{k, j=1} ^{n}=\operatorname{det}\right\| K\left(t_{j}, \theta_{\alpha_{k}}\right) \|_{j, k=1}^{n}>0 \text {, }
$$


since $a<\theta_{\alpha_{1}}<\theta_{\alpha_{2}}<\cdots<\theta_{\alpha_{n}}<b$. This proves (1). To prove (2), we apply the Gram-Schmidt procedure on $\left(\psi_{k}\right)$ to obtain the orthonormal basis $\left(\phi_{k}\right)$ given by

$$
\begin{aligned}
& \dot{\phi}_{1}=\psi_{1} /\left\|\chi_{1}\right\|, \chi_{1}=\psi_{1} \\
& \dot{\phi}_{n}=\chi_{n} /\left\|\chi_{n}\right\|, \chi_{n}=\dot{\psi}_{n}-\sum_{k=1}^{n-1}\left(\psi_{n}, \dot{\phi}_{k}\right) \dot{\phi}_{k}, \quad n=2,3, \cdots .
\end{aligned}
$$

Now it is easily verified that

$$
\operatorname{det}\left\|\phi_{k}\left(t_{j}\right)\right\|_{k, l=1}^{n}=\frac{1}{\left\|\chi_{1}\right\| \cdots\left\|\chi_{n}\right\|} \operatorname{det}\left\|\psi_{k}\left(t_{j}\right)\right\|_{k, j=1}^{n}>0,
$$

for $a \leqq t_{1}<t_{2}<\cdots<t_{n} \leqq b$, since $\left(\psi_{k}\right)_{k=1}^{n}$ is also CT on $[a, b]$. This concludes the proof of the theorem.

Let $t \in D$ and consider the closed convex subsets of $H$

$$
A_{k}(t)=\left\{f \in H: f^{(n)}(t)=\delta_{n k}, n=0,1, \cdots, k\right\}, k=0,1, \cdots .
$$

Since $H$ contains the polynomials these subsets are not empty. Let $\dot{\phi}_{k}(z) \equiv \dot{\phi}_{k}(z ; t)$ be the unique solution of the minimal problem

$$
\lambda_{l}=\lambda_{k}(t)=\min \left\{\|f\|^{2}: f \in A_{k}(t)\right\} .
$$

Clearly, the sequence $\left(\dot{\phi}_{l}\right)_{k=0}^{\infty}$ forms a complete orthogonal system in $H$. Therefore,

$$
K(z, \bar{\zeta})=\sum_{k=0}^{\infty} \psi_{k}(z) \overline{\psi_{k}(\zeta)}, \quad z, \zeta \in D
$$

where

$$
\dot{\psi}_{k}=\dot{\phi}_{k} /\left\|\dot{\phi}_{k}\right\|=\phi_{k} / \lambda_{k}^{1 / 2} \text {. }
$$

Inserting (3) in (2), and using Lemma 5, we obtain, after some manipulations,

$$
K^{*} \overbrace{\left(\begin{array}{l}
z, \cdots, z \\
\bar{t}, \cdots, \bar{t}
\end{array}\right)}^{m}=W\left[\dot{\psi}_{0}(z), \cdots, \psi_{m-1}(z)\right] / \sqrt{\lambda_{0} \lambda_{1} \cdots \lambda_{m-1}} .
$$

We therefore obtain

Theorem 3. $K(z, \bar{t})$ is ETP on $D$ if and only if, for any $t \in D$, the orthonormal basis $\left(\psi_{k}(z ; t)\right)_{k=0}^{\infty}$ is ECT on $D$.

We remark that, for the fixed point $t \in D$, we have

$$
W\left[\psi_{0}(t), \cdots, \dot{\psi}_{m-1}(t)\right]=1 / \sqrt{\lambda_{0} \lambda_{1} \cdots \lambda_{m-1}} .
$$


Thus

$$
K^{*}(\overbrace{\left.\begin{array}{l}
t, \cdots, t \\
\bar{t}, \cdots, \bar{t}
\end{array}\right)}^{m}=1 / \lambda_{0} \lambda_{1} \cdots \lambda_{m-1} .
$$

It is of course clear that the assumption that $H$ contains the polynomials can be replaced by the requirement that for any $t \in D, A_{k}(t) \neq \varnothing, k=0,1, \cdots$. Note also that

$$
\lambda_{0}(t)=1 / K(t, \bar{t})>0,
$$

and moreover the $\lambda$ 's introduced above are connected to certain Gaussian curvatures of Kähler metrics induced by $K(t, \bar{t})$. More explicitly, the Kähler metric $d S^{2}=K(z, \bar{z})|d z|^{2}$ has the Gaussian curvature

$$
C_{S}(z)=-\frac{1}{2} \nabla \log K / K=-\frac{2}{K} \frac{\partial^{2} \log K}{\partial z \partial \bar{z}}, K=K(z, \bar{z}) .
$$

Therefore, using (4) and (5),

$$
C_{S}(t)=-\frac{2}{K^{3}} K^{*}\left(\begin{array}{ll}
t, & t \\
\bar{t}, \bar{t}
\end{array}\right)=-2 \frac{\lambda_{0}^{2}}{\lambda_{1}}<0, K=K(t, \bar{t}), t \in D .
$$

THEOREM 4. Let $D$ be a simply-connected domain and assume that each automorphism $w=w(z)$ induces an isometry $T$ of $H\left(D_{w}\right)$ onto $H\left(D_{z}\right)$, given by $(T f)(z)=f(w(z))\left[w^{\prime}(z)\right]^{\alpha}$. Then

$$
K^{*}(\overbrace{\left.\begin{array}{l}
z, \cdots, z \\
\bar{t}, \cdots, \bar{t}
\end{array}\right)}^{m}=\frac{\lambda_{0}{ }^{m(m+2 \alpha-1) / 2 \alpha}}{\lambda_{0} \lambda_{1} \cdots \lambda_{m-1}}[K(z, \bar{t})]^{m(m+2 \alpha-1) / 2 \alpha},
$$

$$
m=1,2, \cdots,
$$

where the $\lambda_{j}=\lambda_{j}\left(t_{0}\right), j=0,1, \cdots, m-1$, are positive constants and $t_{0}$ is any point of $D$.

Proof. $T: H\left(D_{w}\right) \rightarrow H\left(D_{z}\right)$, (the subscript in $D_{w}$ means that $D$ is described in the $w$-plane), is an isometry of the corresponding Hilbert spaces. Therefore,

$$
K(z, \bar{t})=K(w, \bar{\tau})\left[w^{\prime}(z)\right]^{\alpha}\left[\tau^{\prime}(t)\right]^{\alpha}, \tau=w(t), t \in D .
$$

Inserting (7) in (2) we obtain, after some manipulations

$$
K^{*} \overbrace{\left(\begin{array}{l}
z, \cdots, z \\
\bar{t}, \cdots, \bar{t}
\end{array}\right)}^{m}=\left[w^{\prime}(z)\right]^{m(m+2 \alpha-1) / 2}\left[\overline{\left.\tau^{\prime}(t)\right]}\right]^{m(m+2 \alpha-1) / 2} K^{*}(\overbrace{\left.\begin{array}{l}
w, \cdots, w \\
\bar{\tau}, \cdots, \bar{\tau}
\end{array}\right)}^{m} .
$$


Consider the function

$$
J(z, \bar{t})=K^{*} \overbrace{\left(\begin{array}{l}
z, \cdots, z \\
\bar{t}, \cdots, \bar{t}
\end{array}\right) /[K(z, \bar{t})]^{m(m+2 \alpha-1) / 2 \alpha}}^{m} .
$$

Since $D$ is homogeneous (i.e., the automorphism group of $D$ is transitive) it follows, from (7) and (8) that $J(z, \bar{t})$ is constant in $D$. We evaluate this constant at a fixed point $t_{0} \in D$. According to (4) and (5) $J\left(t_{0}, \bar{t}_{0}\right)=\lambda_{0}^{m(m+2 \alpha-1) / 2 \alpha} /\left(\lambda_{0} \lambda_{1} \cdots \lambda_{m-1}\right)$, where $\lambda_{j}=\lambda_{j}\left(t_{0}\right)$ are positive constants. Therefore (6) is true.

COROLLARY. Under the assumptions of Theorem 4 and if, in addition, $K(z, \bar{t})$ never vanishes in $D$, then $K(z, \bar{t})$ is ETP on $D$.

REMARK. In the case of Theorem $4, d S^{2}=[K(z, \bar{z})]^{1 / \alpha}|d z|^{2}$ is a conformally invariant metric for $\alpha>0$. Therefore, its Gaussian curvature $C_{\alpha}(z)$ is a constant in $D$. We have

$$
\begin{aligned}
C_{\alpha}(z) & =\frac{-2}{K^{1 / \alpha}} \frac{1}{\alpha} \frac{\partial^{2} \log K}{\partial z \partial \bar{z}}=-\frac{2}{\alpha} \frac{1}{K^{(1+2 \alpha) / \alpha}} \cdot K^{*}\left(\begin{array}{l}
z, z \\
\bar{z}, \bar{z}
\end{array}\right) \\
& =-\frac{2}{\alpha} \frac{\lambda_{0}^{(1+2 \alpha) / \alpha}}{\lambda_{0} \lambda_{1}}=-\frac{2}{\alpha} \frac{\lambda_{0}^{(\alpha+1) / \alpha}}{\lambda_{1}}<0 .
\end{aligned}
$$

Therefore,

$$
K^{*}\left(\begin{array}{l}
z, z \\
\bar{t}, \bar{t}
\end{array}\right)=-\frac{\alpha}{2} C_{\alpha}\left(t_{0}\right)[K(z, \bar{t})]^{(2 \alpha+1) / \alpha}
$$

3. The kernels of Hardy spaces. Let $\nu>-1$. The Hardy space $\mathscr{D}_{\nu}$ is defined as the set of all functions $F(z)$, analytic in the upper half plane $U=\{z: \operatorname{Im} z>0\}$, of the form

$$
F(z)=\int_{0}^{\infty} t^{\nu / 2} e^{i z t} f(t) d t
$$

with $f(x)$ in $L_{2}(0, \infty) . \mathscr{D}_{\nu}$ is a Hilbert space ([3], p. 219) with the norm

$$
\|F\|_{\nu}^{2}=\int_{0}^{\infty}|f(t)|^{2} d t
$$

Of course, point evaluation is a bounded functional and, in fact, $|F(z)|^{2} \leqq \Gamma(\nu+1)[2 \operatorname{Im} z]^{-(\nu+1)}\|F\|_{\nu}^{2}$. The sequence

$$
\psi_{k}(z)=\left(\frac{1+i z}{1-i z}\right)^{k}\left(\frac{2}{1-i z}\right)^{1+\nu}, \quad k=0,1, \cdots,
$$


is an orthogonal basis in $\mathscr{D}_{\nu}$ and $\left\|\psi_{k}\right\|_{\nu}^{2}=k ! / \Gamma(1+\nu+k)$. Therefore, the reproducing kernel of $\mathscr{D}_{\nu}$ is given by

$$
K_{\nu}(z, \bar{t})=\sum_{k=0}^{\infty} \frac{\Gamma(1+\nu+k)}{k !} \psi_{k}(z) \overline{\psi_{k}(t)},
$$

or

$$
K_{\nu}(z, \bar{t})=\Gamma(1+\nu) /(i \bar{t}-i z)^{1+\nu} .
$$

(The fractional power is chosen to be continuous in $U$ and positive at $t$.)

Let $w=w(z)$ be an automorphism of $U$ (i.e., $w(z)=(a z+b) /(c z+d)$, $a, b, c, d$ real and $a d-b c>0)$. Then $(T F)(z)=F(w)\left[w^{\prime}\right]^{(\nu+1) / 2}$ is an isometry of $\mathscr{D}_{\nu}$. Since $U$ is simply connected it follows from Theorem 4 that

$$
K_{\nu}^{*}(\overbrace{\left.\begin{array}{l}
z, \cdots, z \\
\bar{t}, \cdots, \bar{t}
\end{array}\right)}^{m}=\frac{\lambda_{0}^{m(m+\nu) /(\nu+1)}}{\lambda_{0} \lambda_{1} \cdots \lambda_{m-1}}\left[K_{\nu}(z, \bar{t})\right]^{m(m+\nu) /(\nu+1)},
$$

$$
m=1,2, \cdots,
$$

where $\lambda_{j}=\lambda_{j}(i)>0$ are evaluated at $i \in U$. Since $K_{\nu}(z, \bar{t})$ is never zero we obtain:

THEOREM 5. $K_{\nu}(z, \bar{t})=\Gamma(1+\nu) /(i \bar{t}-i z)^{1+\nu}, \nu>-1$, is ETP on the upper half plane $U$. Moreover it is strictly ETP (i.e., the Wronskians in (9) are positive) on all straight lines in $U$ which are parallel to the imaginary axis.

REMARK. When $z$ and $t$ are on the imaginary axis, i.e., when $z=i y, t=i r, y>0, r>0$, we have $K_{\nu}(i y,-i r)=\Gamma(1+\nu) /(r+y)^{1+\nu}$. Thus $\Gamma(1+\nu) /(r+y)^{1+\nu}$ is $\operatorname{ETP}$ on $(0, \infty) \times(0, \infty)$ for $\nu>-1$. Especially, when $\nu=0, K_{0}(i y,-i r)=1 /(r+y)$ is the famous Cauchy kernel. The fact that this kernel is ETP is used to establish the wellknown Müntz theorem on best polynomial approximation.

If $U$ is mapped conformally onto the unit disc $\Delta=\{w:|w|<1\}$ the mapping $w(z)=e^{i \theta}(z-\alpha) /(z-\bar{\alpha}), \alpha \in U$, induces the natural isometry of the corresponding Hilbert spaces. Consequently,

$$
K_{\nu}(z, \bar{t})=K_{\nu}(w, \bar{\tau})\left[w^{\prime}(z)\right]^{(\nu+1) / 2}\left[{\overline{\tau^{\prime}(t)}}^{\nu+1 / 2}, \tau=w(t) \in \Delta,\right.
$$

or

$$
K_{\nu}(w, \bar{\tau})=\Gamma(1+\nu) /(1-w \bar{\tau})^{1+\nu}, \quad w, \tau \in \Delta .
$$

Using (10) and Theorem 5 we obtain: 
COROLlaRY. The reproducing kernel

$$
K_{\nu}(z, \bar{t})=\Gamma(1+v)(1-z \bar{t})^{1+\nu}, \nu>-1,
$$

is ETP on $\Delta=\{z:|z|<1\}$. Especially, it is strictly ETP on all diameters of $\Delta$. (Karlin, using Laplace transforms, has also shown (unpublished) that $(1-x y)^{1+\nu}$ is $\operatorname{ETP}$ on $\left.(-1,1) \times(-1,1), \nu>-1\right)$.

We continue to identify these kernels as follows: If $\nu>0$ then for $F \in \mathscr{D}_{\nu}$ it holds that ([3], p. 219);

$$
\|F\|_{\nu}^{2} 2^{-\nu+1} \pi \Gamma(\nu)=\left.\int_{U} \int F(z)\right|^{2}[\operatorname{Im} z]^{\nu-1} d x d y .
$$

If $\nu=0$ then for $F \in \mathscr{D}_{0}$

$$
2 \pi\|F\|_{0}^{2}=\sup _{y>0} \int_{-\infty}^{\infty}|F(x+i y)|^{2} d x .
$$

We again map $U$ onto the unit disc $\Delta$ by $w=e^{i \theta}(z-\alpha) /(z-\bar{\alpha})$, $\alpha \in \Delta . \quad(T f)(z)=f(w)\left[w^{\prime}\right]^{(\nu+1) / 2}$ is an isometry of the Hilbert space of functions on $\Delta$ onto $\mathscr{D}_{\nu}$. But $1-|w|^{2}=2\left|w^{\prime}\right| \cdot \operatorname{Im} z$. Therefore, using (11) and (12), we have

$$
\|T f\|_{\nu}^{2}=\frac{1}{\pi \Gamma(1+\nu)} \int_{4}|f(w)|^{2} \nu\left(1-|w|^{2}\right)^{\nu-1} d u d v, \quad(w=u+i v),
$$

and

$$
\|T f\|_{0}^{2}=\frac{1}{2 \pi} \int_{\partial \Delta}|f(w)|^{2}|d w| .
$$

In the last integral, $f$ stands for the nontangential boundary value of the analytic function $f(w)$ in $\Delta$. Of course, $T^{-1}\left(\mathscr{D}_{0}\right)$ is the HardySzegö space with the reproducing Szegö kernel $1 /(1-w \bar{\tau}), w, \tau \in \Delta$.

We let $H^{(\nu)} \equiv H^{(\nu)}(\Delta)=\pi \Gamma(1+\nu) T^{-1}\left(\mathscr{D}_{\nu}\right)$ for $\nu>0$ and $H^{(0)} \equiv$ $H^{(0)}(\partial \Delta)=2 \pi T^{-1}\left(\mathscr{D}_{0}\right)$. Therefore, $H^{(\nu)}(\Delta), \nu>0$, is the Hilbert space of analytic functions $f(z)$ in the unit disc $\Delta=\{z:|z|<1\}$ with the inner product

$$
(f, g)_{\nu}=\int_{\Delta} \int f(z) \overline{g(z)} \nu\left(1-|z|^{2}\right)^{\nu-1} d x d y, f, g \in H^{(\nu)}, \nu>0 .
$$

Naturally, $H^{\circ}(\partial \Delta)$ is the Hardy-Szegö space with the inner product $(f, g)_{0}=\int_{\partial \Delta} f(z) \overline{g(z)}|d z|$ (the integral is taken over the nontangential boundary values of $f$ and $g$ ).

Each $f \in H^{(\nu)}$ is given by $f(z)=\sum_{n=0}^{\infty} a_{n} z^{n}$ and

$$
\|F\|_{\nu}^{2}=2 \pi \nu \sum_{n=0}^{\infty}\left|a_{n}\right|^{2} \int_{0}^{1}\left(1-r^{2}\right)^{\nu-1} r^{2 n+1} d r, \quad \nu>0 .
$$


Therefore,

$$
\|f\|_{\nu}^{2}=\pi \sum_{n=0}^{\infty}\left(\begin{array}{c}
\nu+n \\
n
\end{array}\right)^{-1}\left|a_{n}\right|^{2}, \quad \nu>0
$$

Similarly,

$$
\|f\|_{0}^{2}=2 \pi \sum_{n=0}^{\infty}\left|\alpha_{n}\right|^{2}
$$

It follows that the sequence $\psi_{n}(z)=\pi^{-1 / 2}\left(\begin{array}{c}\nu+n \\ n\end{array}\right)^{1 / 2} z^{n}, n=0,1, \cdots$, forms an orthonormal basis for $H^{(\nu)}$ and $\left(z^{n} / \sqrt{2 \pi}\right)_{0}^{\infty}$ is an orthonormal basis for $H^{(0)}$. Therefore

$$
K_{\nu}(z, \bar{t})=\frac{1}{\pi} \frac{1}{(1-z \bar{t})^{\nu+1}}, \quad z, t \in \Delta
$$

is the reproducing kernel of $H^{(\nu)}, \nu>0$, and

$$
K_{0}(z, \bar{t})=\frac{1}{2 \pi} \frac{1}{1-\mathrm{z} \bar{t}}, \quad z, t \in \Delta,
$$

is the reproducing kernel of $H^{(0)}$.

We have shown that $1 /(1-z \bar{t})^{\nu+1}, \nu>-1$, is ETP on $\Delta$. For $\nu>0$, however this fact can be shown directly from the definition of $H^{(\nu)}$. Indeed for any $w=w(z), w(z)=e^{i \theta}\left(z-t_{0}\right) /\left(1-z \bar{t}_{0}\right), t_{0} \in \Delta$, $(T f)(z)=f(w)\left[w^{\prime}\right]^{(\nu+1) / 2}$ is an isometry of the corresponding Hilbert spaces. Therefore, again,

$$
\begin{array}{r}
K_{\nu}^{*} \overbrace{\left(\begin{array}{l}
z, \cdots, z \\
\bar{t}, \cdots, \bar{t}
\end{array}\right)}^{m}=\frac{\lambda_{0}^{m(m+\nu)(\nu+1)}}{\lambda_{0} \lambda_{1} \cdots \lambda_{m-1}}\left[K_{\nu}(z, \bar{t})\right]^{(m(m+\nu)) /(\nu+1)}, \\
m=1,2, \cdots,
\end{array}
$$

where $\lambda_{j}=\lambda_{j}(0)>0$ are evaluated at $0 \in \Delta$ and $K_{2}(z, \bar{t})$ is the reproducing kernel of $H^{(\nu)}, \nu \geqq 0$. Now, $\phi_{k}(z ; 0)=z^{k} / k !, k=0,1, \cdots$, are the solutions for the minimal problems related to $\lambda_{k}(0)$, thus

$$
\lambda_{k}(0)=\frac{1}{(k !)^{2}}\left\|z^{k}\right\|_{\nu}^{2}=\frac{\pi}{(k !)^{2}}\left(\begin{array}{c}
\nu+k \\
k
\end{array}\right)^{-1}=\frac{\pi}{k !} \frac{\Gamma(\nu+1)}{\Gamma(\nu+k+1)},
$$

$k=0,1, \cdots$. From the fact that the positive quantity

$$
\lambda_{0}^{(m(m+\nu)) /(\nu+1)} / \lambda_{0} \lambda_{1} \cdots \lambda_{m-1}
$$

is conformally invariant we obtain:

Theorem 6. Let $L_{\Delta}(z, \bar{t})=1 /(1-z \bar{t})^{\nu+1}, z, t \in \Delta$, and $L_{U}(z, \bar{t})=$ 


$$
\begin{aligned}
& 1 /(i \bar{t}-i z)^{\nu+1}, z, t \in U, \nu>-1 \text {. Then } \\
& L_{\Delta}^{*}(\overbrace{\left.\begin{array}{l}
z, \cdots, z \\
\bar{t}, \cdots, \bar{t}
\end{array}\right)}^{m}=\frac{1}{[\Gamma(\nu+1)]^{m}} \prod_{k=0}^{m-1}[k ! \Gamma(\nu+k+1)]\left[L_{\Delta}(z, \bar{t})\right]^{m(m+\nu) /(\nu+1)},
\end{aligned}
$$

and

$$
\begin{aligned}
& L_{U}^{*}(\overbrace{\left(\begin{array}{l}
z, \cdots, z \\
\bar{t}, \cdots, \bar{t}
\end{array}\right)}^{m}=\frac{1}{[\Gamma(\nu+1)]^{m}} \prod_{k=0}^{m-1}[k ! \Gamma(\nu+k+1)] \cdot\left[L_{U}(z, \bar{t})\right]^{m(m+\nu) /(\nu+1)}, \\
& m=1,2, \cdots \text {. }
\end{aligned}
$$

4. The Bergman and Szegö kernel functions. Let $D$ be a plane domain whose boundary components do not reduce to a point and consider the Bergman space $H^{(1)} \equiv H^{(1)}(D) . \quad H^{(1)}(D)$ is the set of all analytic functions in $D$ with a single-valued primitive in $D$ normed by $\|f\|_{1}^{2}=\int_{\Delta} \int|f(z)|^{2} d x d y, f \in H^{(1)}$. $H^{(1)}$ is a Hilbert space with the Bergman reproducing kernel $K_{D}(z, \bar{t})$ ([2], p. 49).

Let $w: D \rightarrow D^{*}$ be a conformal mapping of $D$ onto $D^{*}$. Then $(T f)(z)=f(w) w^{\prime}$ is an isometry of $H^{(1)}\left(D^{*}\right)$ onto $H^{(1)}(D)$. Therefore,

$$
K_{D}(z, \bar{t})=K_{D^{*}}(w, \bar{\tau}) w^{\prime}(z) \overline{\tau^{\prime}(t)}, \tau=w(t) \in D^{*} .
$$

THEOREM 7. Let $N_{D}$ be the connectivity of D. $K_{D}(z, \bar{t})$ is ETP on $D$ if and only if $N_{D}=1$.

Proof. According to (15) we can assume that $D$ is bounded by $N_{D}$ analytic curves. We use the identity ([2], p. 100) connecting $K_{D}(z, \bar{t})$ with the slit mapping. From the above mentioned identity follows that for each fixed $t \in D, K_{D}(z, \bar{t})$ has $2\left(N_{D}-1\right)$ zeros (counting multiplicities) in $D$ and none near $\partial D$. Consequently, if $K_{D}(z, \bar{t})$ is ETP on $D$ then $N_{D}=1$. Conversely, let $N_{D}=1$. Clearly, by (15),

$$
J_{D}(z, \bar{t})=K_{D}^{*}(\overbrace{\left(\begin{array}{ll}
z, \cdots, z \\
\bar{t}, \cdots, \bar{t}
\end{array}\right)}^{m} /\left[K_{D}(z, \bar{t})\right]^{m(m+1) / 2}
$$

is conformally invariant. Since $D$ is simply-connected it follows by the Riemann mapping theorem that $J_{D}(z, \bar{t})$ is a constant for all simply-connected domains. Therefore,

$$
J_{D}(z, \bar{t})=J_{\Delta}(0,0), \quad \Delta=\{w:|w|<1\},
$$

where as before 


$$
J_{\Delta}(0,0)=\lambda_{0}^{(m(m+1) / 2} /\left(\lambda_{0} \lambda_{1} \cdots \lambda_{m-1}\right),
$$

and

$$
\lambda_{k}=\lambda_{k}(0)=\frac{\pi}{(k+1)(k !)^{2}}, \quad k=0,1, \cdots
$$

Therefore,

$$
K_{D}^{*}(\overbrace{\left.\begin{array}{l}
z, \cdots, z \\
\bar{t}, \cdots, \bar{t}
\end{array}\right)}^{m}=m ! \pi^{m(m-1) / 2}\left(\prod_{k=0}^{m-1} k !\right)^{2}\left[K_{D}(z, \bar{t})\right]^{(m(m+1)) / 2} .
$$

According to (13) $K_{\Delta}(w, \bar{\tau})=K_{1}(w, \bar{\tau})=\pi^{-1} /(1-w \bar{\tau})^{2}$. Hence, using (15) and $(16), K_{D}(z, \bar{t})$ is $\operatorname{ETP}$ on $D$.

REMARK. $J_{\Delta}(0,0)$ are the Gaussian curvatures related to the Poincaré metric

$$
d S_{\Delta}^{2}=\pi^{-1}\left(1-|z|^{2}\right)^{-2}|d z|^{2} .
$$

The Gaussian curvature of this metric is $-2 J_{\Delta}(0,0)$, where $m=2$. Thus, $-2 J_{\Delta}(0,0)=-4 \pi$. (Compare [2], p. 38.)

The Hardy-Szegö space $H^{(0)} \equiv H^{(0)}(\partial D)$ is the set of all analytic functions $f(z)$ in $D$ that possess well-behaved nontangential boundary values $f(\zeta), \zeta \in \partial D$, in $L_{2}(\partial D)$. The norm is the $L_{2}(D)$ norm, i.e., $\|f\|_{0}^{2}=\int_{\partial D}|f(\zeta)|^{2}|d \zeta| . \quad H^{(0)}$ is a Hilbert space with the Szegö reproducing kernel $\hat{K}_{D}(z, \bar{t})([2]$, p. 108). Similarly to (15), we have, under conformal mappings,

$$
\left[\hat{K}_{D}(z, \bar{t})\right]^{2}=\left[\hat{K}_{D^{*}}(w, \bar{\tau})\right]^{2} w^{\prime}(z) \overline{\tau^{\prime}(t)}, \tau=w(t) \in D^{*},
$$

(see also [4]).

Similarly to Theorem 7 we have

THEOREM 8. $\hat{K}_{D}(z, \bar{t})$ is ETP on $D$ if and only if $N_{D}=1$.

Proof. This follows from (14), (17) and the fact $\hat{K}_{D}(z, \bar{t})$ has (for fixed $t \in D) N_{D}-1$ zeroes (counting multiplicities) in $D$ and none near $\partial D,([2]$, p. 118).

In analogy to (16) we have ( $D$ is simply-connected)

$$
\hat{K}_{D}^{*} \overbrace{\left(\begin{array}{l}
z, \cdots, z \\
\bar{t}, \cdots, \bar{t}
\end{array}\right)}^{m}=(2 \pi)^{m(m-1)}\left(\sum_{k=0}^{m-1} k !\right)^{2}\left[\hat{K}_{D}(z, \bar{t}]^{m^{2}}\right.
$$


The following is a generalization of Theorem 4 .

THEOREM 9. Let $D$ be simply-connected and assume that each automorphism $w=w(z)$ of $D$ induces an isometry $T$ of $H\left(D_{w}\right)$ onto $H\left(D_{z}\right)$, given by $(T f)(z)=f(w) \cdot G(w), f \in H\left(D_{w}\right)$. Then

$$
K^{*}\left(\begin{array}{l}
m, \cdots, z \\
\bar{t}, \cdots, \bar{t}
\end{array}\right)=\frac{\lambda_{0}^{m}}{\lambda_{0} \lambda_{1} \cdots \lambda_{m-1}}\left[\frac{K_{D}(z, \bar{t})}{K_{D}\left(t_{0}, \bar{t}_{0}\right)}\right]^{(m(m-1) / 2} \cdot[K(z, \bar{t})]^{m}
$$

where the $\lambda_{j}=\lambda_{j}\left(t_{0}\right)>0, t_{0} \in D$, are related to the space $H=H(D)$ with the reproducing kernel $K(z, \bar{t})$ and $K_{D}(z, \bar{t})$ is the Bergman kernel. Especially, if $K(z, \bar{t})$ never vanishes in $D$ then it is ETP on $D$.

Proof. This follows immediately from (15), from

$$
K(z, \bar{t})=K(w, \bar{\tau}) G(w) \overline{G(\tau)}, \tau=w(t) \in D^{*}
$$

and from

$$
K^{*}(\overbrace{\left.\begin{array}{l}
z, \cdots, z \\
\bar{t}, \cdots, \bar{t}
\end{array}\right)}^{m}=[G(w) \overline{G(\tau)}]]^{m}\left[w^{\prime}(z) \bar{\tau}^{\prime}(t)\right]^{m(m-1) / 2} K^{*}\left(\begin{array}{l}
w, \cdots, w \\
\bar{\tau}, \cdots, \bar{\tau}
\end{array}\right) .
$$

CoRollary. Let $D$ be simply-connected and let $\rho(z), 1 / \rho(z)$ be analytic functions in $D$. Consider the Hilbert space $H_{\rho}^{(0)}$ and $H_{\rho}^{(1)}$ of analytic functions $f(z)$ in $D$ normed by

$$
\|f\|_{0}^{2}=\int_{\partial D}|\rho(z)|^{-2}|f(z)|^{2}|d z| \text { and }\|f\|_{3}^{2}=\int_{D} \int|\rho(z)|^{-2}|f(z)|^{2} d x d y
$$

respectively. Then the reproducing kernels of $H_{\rho}^{(0)}$ and $H_{\rho}^{(1)}$ are ETP on D. Moreover, $\hat{K}_{\rho}(z, \bar{t})=\hat{K}_{D}(z, \bar{t}) \rho(z) \overline{\rho(t)}$ is the kernel of $H_{\rho}^{(0)}$ and $K_{\rho}(z, \bar{t})=K_{D}(z, \bar{t}) \rho(z) \overline{\rho(t)}$ is the kernel of $H_{\rho}^{(1)}$.

For $\nu>0$ we can generalize $H^{(\nu)}(\Delta)$ as follows: $H^{(\nu)}(D)$ is the Hilbert space of all analytic functions $f(z)$ in $D$ with a single-valued primitive in $D$ and normed by

$$
\|f\|_{\nu}^{2}=\left.\frac{\nu}{\pi^{(\nu-1) / 2}} \int_{D} \int f(z)\right|^{2}\left[K_{D}(z, \bar{z})\right]^{(1-\nu) / 2} d x d y .
$$

Of course, when $\nu=1$ this is the usual Bergman space. Let $K_{D_{\nu}}(z, \bar{t})$ be the reproducing kernel of $H^{(\nu)}(D)$. Assume now that $D$ is simplyconnected. If $w: D \rightarrow D^{*}$ is conformal, then $(T f)(z)=f(w)\left[w^{\prime}\right]^{(\nu+1) / 2}$ is an isometry of $H^{(\nu)}\left(D^{*}\right)$ onto $H^{(\nu)}(D)$. This is because 


$$
K_{D}(z, \bar{z})=K_{D} *(w, \bar{w})\left|w^{\prime}\right|^{2}
$$

Therefore,

$$
K_{D_{\nu}}(z, \bar{t})=K_{D_{\nu}} *(w, \bar{\tau})\left[w^{\prime}(z)\right]^{(\nu+1) / 2}\left[\overline{\tau^{\prime}(t)}\right]^{(\nu+1) / 2}, \tau=w(t) \in D^{*} .
$$

Especially, when $D^{*}=\Delta=\{w:|w|<1\}$,

$$
K_{D_{\nu}}(z, \bar{t})=\frac{1}{\pi} \frac{1}{(1-w \bar{\tau})^{\nu+1}}\left[w^{\prime} \bar{\tau}^{\prime}\right]^{(\nu+1) / 2} .
$$

Therefore, $K_{D_{\nu}}(z, \bar{t})$ is ETP on $D$ and

$$
K_{D_{\nu}}^{*}(\overbrace{\left(\begin{array}{l}
z, \cdots, z \\
\bar{t}, \cdots, \bar{t}
\end{array}\right)}^{m}=\frac{\pi^{(m(m-1) / \nu+1}}{[\Gamma(\nu+1)]^{m}} \prod_{k=0}^{m-1}[k ! \Gamma(\nu+k+1)] \cdot\left[K_{D_{\nu}}(z, \bar{t})\right]^{m(m+\nu) / \nu+1} .
$$

Example. Consider the oval of Cassini,

$$
C=\left\{z: \operatorname{Rez}>0,\left|z^{2}-1\right|<r, r<1\right\} .
$$

This is a simply-connected domain of four-fold symmetry (i.e., if $z \in C$ then $-z, \bar{z} \in C) . \quad w=\left(z^{2}-1\right) / r$ maps $C$ onto $\triangle$ conformally. Therefore,

$$
K_{C_{\nu}}(z, \bar{t})=\frac{(2 r)^{\nu+1}}{\pi} \frac{(z \bar{t})^{(\nu+1) / 2}}{\left[r^{2}-\left(z^{2}-1\right)\left(\bar{t}^{2}-1\right)\right]^{2+1}},
$$

is the reproducing kernel of $H^{(\nu)}(C)$. Consequently, $K_{C_{\nu}}(z, \bar{t})$ is ETP on $C$. Especially, it is strictly ETP on all diameters of $C$. Therefore, $(x y)^{(\nu+1) / 2} /\left[r^{2}-\left(x^{2}-1\right)\left(y^{2}-1\right)\right]^{\nu+1}, \nu>-1$, is strictly ETP on $(\sqrt{1-r}$, $\sqrt{1+r}), 0<r<1$.

5. The exponential kernel. Let $H=H(C)$ be the space of entire functions $f(z)$ normed by

$$
\|f\|^{2}=\frac{1}{\pi} \int_{C} \int|f(z)|^{2} e^{-\mid z i^{2}} d x d y .
$$

Let

$$
M_{f}(r)=\frac{1}{\pi} \int_{|z| \leqq r} \int|f(z)|^{2} e^{-|z|^{2}} d x d y, \quad 0<r<\infty .
$$

Then $\|f\|^{2}=\lim _{r \rightarrow \infty} M_{f}(r)$. Each $f \in H$ is given by $f(z)=\sum_{n=0}^{\infty} a_{n} z^{n}$ and thus

$$
M_{f}(r)=2 \sum_{n=0}^{\infty}\left|a_{n}\right|^{2} \int_{0}^{r} \rho^{2 n+1} e^{-\rho^{2}} d \rho
$$


Let

$$
\alpha_{n}(r)=\frac{2}{n !} \int_{0}^{r} \rho^{2 n+1} e^{-\rho^{2}} d \rho, \quad n=0,1, \cdots
$$

Since

$$
\int_{0}^{\infty} \rho^{2 n+1} e^{-\rho^{2}} d \rho=\frac{1}{2} n !
$$

it follows that

$$
M_{f}(r)=\sum_{n=0}^{\infty} n !\left|a_{n}\right|^{2} \alpha_{n}(r)
$$

where $0<\alpha_{n}(r)<1$ and $\lim _{r \rightarrow \infty} \alpha_{n}(r)=1$. From this, it follows (an application of Fatou's lemma) that

$$
(f, f)=\sum_{n=0}^{\infty} n !\left|a_{n}\right|^{2} .
$$

Using the Cauchy-Schwarz inequality we obtain

$$
|f(z)|^{2} \leqq\left(\sum_{n=0}^{\infty} n !\left|a_{n}\right|^{2}\right) \cdot\left(\sum_{n=0}^{\infty} \frac{\left|z^{n}\right|^{2}}{n !}\right) \cdot
$$

Hence

$$
|f(z)| \leqq e^{|z|^{2} / 2}\|f\|
$$

thus, $H$ is a Hilbert space with the reproducing kernel $K(z, \bar{t})$. The simplest orthonormal system in $H$ is $\psi_{n}(z)=z^{n} /(n !)^{1 / 2}, n=0,1, \cdots$, and, by (18), it is also complete. Therefore,

$$
K(z, \bar{t})=e^{z \bar{t}} .
$$

The subgroup of automorphisms of $C$ of the form $w=e^{i \theta} z+B$, $B \in C$, is transitive and induces an isometry of the corresponding Hilbert spaces of the form

$$
(T f)(z)=e^{i \theta} e^{|B|^{2} / 2} e^{-\bar{B} w} f(w) .
$$

Therefore,

$$
e^{z \bar{t}}=A e^{-\bar{B} w-B \bar{\tau}} e^{w \bar{\tau}}, A=e^{|B|^{2}}, \tau=w(t),
$$

and so

$$
K^{*}(\overbrace{\left(\begin{array}{l}
z, \cdots, z \\
\bar{t}, \cdots, \bar{t}
\end{array}\right)}^{m}=A^{m} e^{(-\bar{B} w-B \bar{\tau}) m} K^{*}\left(\begin{array}{l}
\overbrace{w, \cdots, w} \\
\bar{\tau}, \cdots, \bar{\tau}
\end{array}\right), \quad m=1,2, \cdots
$$


Consequently, $J(z, \bar{t})=K^{*}(\overbrace{\left.\begin{array}{l}z, \cdots, z \\ \bar{t}, \cdots, \bar{t}\end{array}\right)}^{m}[K(z, \bar{t})]^{m}$ is a constant, say $J(z, \bar{t})=$ $J(0,0)$. Here

$$
J(0,0)=\lambda_{0_{1}}^{m} /\left(\lambda_{0} \lambda_{1} \cdots \lambda_{m-1}\right),
$$

where

$$
\lambda_{k}=\lambda_{k}(0)=\left\|\frac{z^{k}}{k !}\right\|^{2}=1 / k !, \quad k=0,1, \cdots
$$

Therefore,

THEOREM 10. The reproducing kernel $K(z, \bar{t})=e^{z \bar{t}}$ is ETP on $C$ and

$$
K^{*}(\overbrace{\left.\begin{array}{l}
z, \cdots, z \\
\bar{t}, \cdots, \bar{t}
\end{array}\right)}^{m}=\left(\prod_{k=2}^{m-1} k !\right) e^{z \bar{t} m}, \quad m=1,2, \cdots .
$$

It is strictly ETP on all rays passing through the origin. Especially, the well-known result ([6], p. 99), $e^{x y}$ is ETP on $(-\infty, \infty) \times(-\infty, \infty)$.

\section{REFERENCES}

1. N. Aronszajn, Theory of reproducing kernels, Trans. Amer. Math. Soc., 68 (1950), 337-404.

2. S. Bergman, The Kernel Function and Conformal Mapping, Amer. Math. Soc., Math. Surveys 5, Providence, R. I., 1970.

3. L. de Branges, Hilbert Spaces of Entire Functions, Prentice-Hall, Englewood Cliffs, N. J., 1968.

4. J. Burbea, On the Gaussian curvature related to the Szegö metric, (to appear).

5. P. J. Davis, Interpolation and Approximation, Blaisdell, Waltham, Mass., 1963.

6. S. Karlin, Total Positivity, Vol. 1, Stanford Univ. Press, Stanford, Cal., 1968.

7. — Best quadrature formulas and splines, J. Approximation Theory, 4 (1971), 59-90.

8. N. Richter-Dyn, Properties of minimal integration rules, II, SIAM J. Numer. Anal., 8 (1971), 497-508.

Received May 24, 1974. This work is supported by the U. S. Army Grant No. 2065.

Tel-Aviv University, ISRAEL

AND

Pennsylvania State University 



\section{PACIFIC JOURNAL OF MATHEMATICS}

\section{EDITORS}

RICHARD ARENS (Managing Editor)

University of California

Los Angeles, California 90024

\section{R. A. Beaumont \\ University of Washington \\ Seattle, Washington 98105}

\section{J. DugundJI}

Department of Mathematics

University of Southern California

Los Angeles, California 90007

D. Gilbarg and J. Milgram

Stanford University

Stanford, California 94305

\section{ASSOCIATE EDITORS}
E. F. BECKENBACH
B. H. NeumanN
F. WOLF
K. YOSHIDA

\section{SUPPORTING INSTITUTIONS}

UNIVERSITY OF BRITISH COLUMBIA
CALIFORNIA INSTITUTE OF TECHNOLOGY
UNIVERSITY OF CALIFORNIA
MONTANA STATE UNIVERSITY
UNIVERSITY OF NEVADA
NEW MEXICO STATE UNIVERSITY
OREGON STATE UNIVERSITY
UNIVERSITY OF OREGON
OSAKA UNIVERSITY

UNIVERSITY OF BRITISH COLUMBIA

UNIVERSITY OF CALIFORNIA

MONTANA STATE UNIVERSITY

NEW MEXICO STATE UNIVERSITY

OREGON STATE UNIVERSITY

OSAKA UNIVERSITY

\author{
UNIVERSITY OF SOUTHERN CALIFORNIA \\ STANFORD UNIVERSITY \\ UNIVERSITY OF TOKYO \\ UNIVERSITY OF UTAH \\ WASHINGTON STATE UNIVERSITY \\ UNIVERSITY OF WASHINGTON \\ AMERICAN MATHEMATICAL SOCIETY \\ NAVAL WEAPONS CENTER
}

The Supporting Institutions listed above contribute to the cost of publication of this Journal, but they are not owners or publishers and have no responsibility for its content or policies.

Mathematical papers intended for publication in the Pacific Journal of Mathematics should be in typed form or offset-reproduced, (not dittoed), double spaced with large margins. Underline Greek letters in red, German in green, and script in blue. The first paragraph or two must be capable of being used separately as a synopsis of the entire paper. Items of the bibliography should not be cited there unless absolutely necessary, in which case they must be identified by author and Journal, rather than by item number. Manuscripts, in triplicate, may be sent to any one of the editors. Please classify according to the scheme of Math. Reviews, Index to Vol. 39. All other communications should be addressed to the managing editor, or Elaine Barth, University of California, Los Angeles, California, 90024.

The Pacific Journal of Mathematics expects the author's institution to pay page charges, and reserves the right to delay publication for nonpayment of charges in case of financial emergency.

100 reprints are provided free for each article, only if page charges have been substantially paid. Additional copies may be obtained at cost in multiples of 50 .

The Pacific Journal of Mathematics is issued monthly as of January 1966. Regular subscription rate: $\$ 72.00$ a year (6 Vols., 12 issues). Special rate: $\$ 36.00$ a year to individual members of supporting institutions.

Subscriptions, orders for back numbers, and changes of address should be sent to Pacific Journal of Mathematics, 103 Highland Boulevard, Berkeley, California, 94708.

PUBLISHED BY PACIFIC JOURNAL OF MATHEMATICS, A NON-PROFIT CORPORATION

Printed at Kokusai Bunken Insatsusha (International Academic Printing Co., Ltd.), 270, 3-chome Totsuka-cho, Shinjuku-ku, Tokyo 160, Japan.

Copyright (C) 1973 by Pacific Journal of Mathematics Manufactured and first issued in Japan 


\section{Pacific Journal of Mathematics \\ Vol. 55, No. $2 \quad$ October, 1974}

Walter Allegretto, On the equivalence of two types of oscillation for elliptic

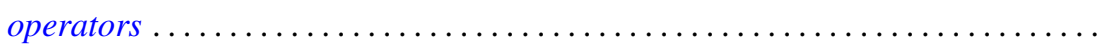

Edward Arthur Bertram, A density theorem on the number of conjugacy classes in

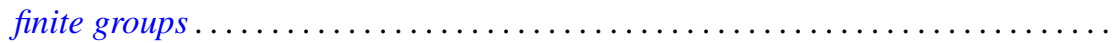

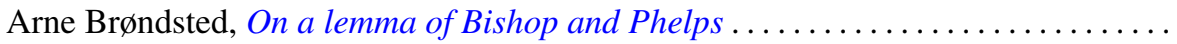

Jacob Burbea, Total positivity and reproducing kernels ..................

Ed Dubinsky, Linear Pincherle sequences . . . . . . . . . . . . . . . . . .

Benny Dan Evans, Cyclic amalgamations of residually finite groups .............

361

Barry J. Gardner and Patrick Noble Stewart, A "going down" theorem for certain

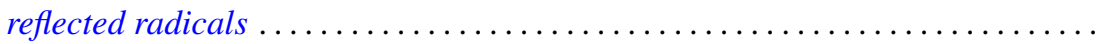

381

Jonathan Light Gross and Thomas William Tucker, Quotients of complete graphs:

revisiting the Heawood map-coloring problem ....................

Sav Roman Harasymiv, Groups of matrices acting on distribution spaces .........

Robert Winship Heath and David John Lutzer, Dugundji extension theorems for

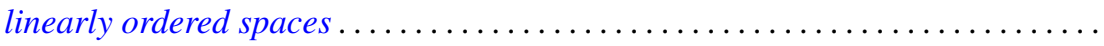

Chung-Wu Ho, Deforming p. l. homeomorphisms on a convex polygonal

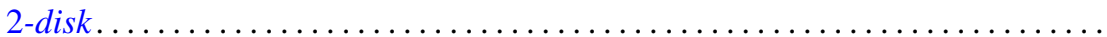

Richard Earl Hodel, Metrizability of topological spaces .................

Wilfried Imrich and Mark E. Watkins, On graphical regular representations of

cyclic extensions of groups .......................... 461

Jozef Krasinkiewicz, Remark on mappings not raising dimension of curves ..... . 479

Melven Robert Krom, Infinite games and special Baire space extensions . . . . . . 483

S. Leela, Stability of measure differential equations . . . . . . . . . . . . . . . . 489

M. H. Lim, Linear transformations on symmetric spaces . . . . . . . . . . . . . . . 499

Teng-Sun Liu, Arnoud C. M. van Rooij and Ju-Kwei Wang, On some group algebra modules related to Wiener's algebra $M_{1} \ldots \ldots \ldots \ldots \ldots \ldots \ldots \ldots \ldots \ldots \ldots$

Dale Wayne Myers, The back-and-forth isomorphism construction ............ 521

Donovan Harold Van Osdol, Extensions of sheaves of commutative algebras by

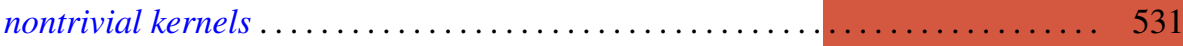

Alan Rahilly, Generalized Hall planes of even order ................... 543

Joylyn Newberry Reed, On completeness and semicompleteness of first countable

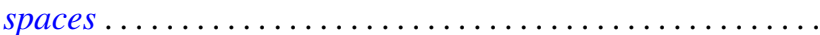

Alan Schwartz, Generalized convolutions and positive definite functions associated

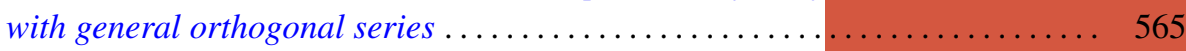

Thomas Jerome Scott, Monotonic permutations of chains . . . . . . . . . . . 583

Eivind Stensholt, An application of Steinberg's construction of twisted groups .... 595

Yasuji Takeuchi, On strongly radicial extensions . . . . ................. 619

William P. Ziemer, Some remarks on harmonic measure in space . . . . . . . . . . 629

John Grant, Corrections to: “Automorphisms definable by formulas” . . . . . . . . 639

Peter Michael Rosenthal, Corrections to: "On an inversion for the general

Mehler-Fock transform pair" ......................... 640

Carl Clifton Faith, Corrections to: "When are proper cyclics injective” . . . . . . 640 